\author{
Urs Fischer \\ Irene Ledermann \\ Krassen Nedeltchev \\ Niklaus Meier \\ Jan Gralla \\ Matthias Sturzenegger \\ Heinrich P. Mattle \\ Marcel Arnold
}

\section{Quality of life in survivors after cervical artery dissection}

Received: 7 April 2008

Received in revised form:

13 September 2008

Accepted: 24 September 2008

Published online: 18 March 2009
U. Fischer, MD • I. Ledermann, MS •

K. Nedeltchev, MD - N. Meier, MD •

M. Sturzenegger, MD - H. P. Mattle, MD .

M. Arnold, MD (四)

Dept. of Neurology, Inselspital

University Hospital Bern and

University of Bern

Freiburgstrasse 4

3010 Bern, Switzerland

Tel.: +41-31/632-1072

Fax: +41-31/632-0321

E-Mail: marcel.arnold@insel.ch

J. Gralla, MD, MSc

Dept. of Neuroradiology, Inselspital

University Hospital Bern and

University of Bern

Bern, Switzerland
Abstract Background and purpose Little data exists about longterm outcome, quality of life (QOL) and its predictors after spontaneous cervical artery dissections (sCAD). Methods Clinical and radiological data of 114 patients with sCAD were collected prospectively. Six patients died within 3 months, the remaining 108 were contacted after a mean of 1498 days (range: 379-3455), 99 survivors (92\%) replied. QOL, assessed with the stroke-specific QOL scale (SSQOL), and functional abilities, measured with modified Rankin Scale (mRS) were compared, and predictors of QOL were analyzed. Subgroup analyses were performed for patients with ischemic stroke, those with isolated local symptoms or transient ischemic symptoms and those without significant disabilities (mRS 0-1) at follow-up. Results Seventy-one of 99 patients $(72 \%)$ had no significant disability, but only $53(54 \%)$ reported a good QOL (SS-QOL $\geq 4$ ). Compared to the self-rated premorbid QOL of all patients, SS-QOL was impaired after sCAD ( $\mathrm{p}<0.001)$; impairment of QOL was observed in patients with ischemic stroke ( $p<0.001)$, in patients with isolated local or transient ischemic symptoms $(\mathrm{p}<0.038)$ and those without significant disabilities at follow-up $(\mathrm{p}=0.013)$. Nevertheless, low mRS was associated with better overall QOL (Kendall's tau >0.5). High National Institute of Health Stroke Scale score on admission and higher age were independent predictors of impaired QOL $(\mathrm{p}<0.05)$. Conclusion QOL is impaired in almost half of long-term survivors after SCAD, even in patients with local or transient symptoms or without functional disability. Impairment of QOL is a surprisingly frequent long-term sequela after SCAD and deserves attention as an outcome measure in these patients.

Key words cervical artery dissection - quality of life - outcome research

\section{Introduction}

Spontaneous cervical artery dissection (sCAD) is a wellrecognized cause of stroke, especially in young patients, with a wide spectrum of clinical presentations $[1,2]$. $\mathrm{Pa}$ tients may present with isolated local manifestations, ischemic signs or both [3]. However, the most appropri- ate end point in follow-up studies remains unclear: several studies used functional outcome, measured with the modified Rankin Scale score (mRS) or the recurrence rate. But the recurrence rate is low and an excellent outcome measured with the mRS is seen in the majority of these patients in hospital- and population-based studies $[4,5]$. But since the mRS captures only functional disability, it may not be sensitive enough to assess the se- 
quelae in patients with $\mathrm{SCAD}$, especially those with transient or local symptoms and patients with no functional disability at follow-up.

QOL scales measuring patient centered outcome are sensitive for minimal changes and do not focus on gross physical aspects of disability only, but include psychological and cognitive functions like memory, emotion, thinking, communication and social role, which may be impaired in patients who have suffered a potentially lifethreatening event such as sCAD.

The aim of the present study was therefore to determine QOL, functional dependency and social status in survivors after sCAD. We analyzed whether QOL was impaired compared to the premorbid condition in all patients and in subgroups (patients with ischemic stroke, those with isolated local symptoms or transient ischemic symptoms and those without significant disabilities (mRS 0-1) at follow-up). In addition, we analyzed whether baseline data on hospital admission predict QOL at long-term follow-up.

\section{Methods}

\section{Investigations}

We prospectively collected data at our stroke center on consecutive patients, presenting with first-ever sCAD from January 1997 to September 2005. Patients with a cervical artery dissection due to major trauma were excluded from this study. All patients underwent a neurological examination, routine blood examination, electrocardiography, magnetic resonance imaging (MRI) of the brain, cervical MRI with T1 fat suppression and magnetic resonance angiography (MRA) or digital subtraction angiography of the four cervical arteries. Internal carotid (ICAD) and/or vertebral artery (VAD) dissection was considered proven if the affected vessel showed an intramural hematoma on axial cervical MRI cuts, or a string sign, intimal flap or pseudoaneurysm on angiography [6-8].

\section{Classification and risk factors}

A sCAD was classified as spontaneous when occurring spontaneously or secondary to minimal trauma [9]. Dissections occurring after obvious head or neck trauma were classified as traumatic and were excluded from the study. sCAD were differentiated into those with and without focal ischemic deficits of the brain, retina, or both. Ischemic deficits were classified according to their duration as stroke $(>24$ hours), transient ischemic attack (TIA; $\leq 24$ hours). The following local signs and symptoms were assessed: headache, neck pain, Horner's syndrome, pulsatile tinnitus and cranial nerve palsy located on the side of dissection. Risk factors for ischemic stroke and SCAD were assessed as reported previously [10]. Arterial hypertension was defined as a positive history of treated or untreated hypertension. A history of migraine with or without aura was diagnosed by a neurologist based on the International Headache Society's criteria [11, 12]. The neurological deficit on admission was graded in all patients using the National Institute of Health Stroke Scale (NIHSS) score [13].

\section{Treatment}

Patients with extracranial SCAD were treated with intravenous heparin, followed by oral warfarin with an international target normalized ratio of 2.5 (range 2.0-3.0) for 3-6 months, unless the patient suffered a hemispheric infarction with a high risk of secondary hemorrhage. Patients with large infarcts or with intracranial extension of a spontaneous VAD received aspirin $100-300 \mathrm{mg} / \mathrm{d}$ for 3-6 months, if no subarachnoid hemorrhage was present on computed tomography or MRI.

\section{QOL and functional outcome measurement}

QOL was assessed with the stroke specific quality of life scale (SSQOL) [14]. The SS-QOL is a disease-specific QOL scale, assessing 12 domains (energy, family roles, language, mobility, mood, personality, self-care, social roles, thinking, upper extremity function, vision and work) (Link to the questionnaire: http://www.strokecenter.org/trials/ scales/ssqol.html). Each item is ranked on a 5-point Likert scale, with higher scores indicating better function. Domain scores are unweighted averages of the items compromising that domain, and the summary SS-QOL is an unweighted average of the 12 domain scores. A summary SS-QOL score of $\geq 4$ was considered to indicate good QOL, $<4$ an impaired QOL. Functional outcome was measured using the mRS [15]. Favorable outcome was defined as mRS 0-1, unfavorable outcome as mRS 2 to 6 .

\section{Follow-up}

Functional outcome and QOL were assessed from February 2006 to March 2007. If a patient was not able to communicate via phone, e.g., because of aphasia, a caregiver was contacted. In a structured telephone interview mRS, residential status, occupation and marital status were assessed [16]. QOL was assessed using the SS-QOL questionnaire, which was sent to patients after the phone contact. They were asked to rate their QOL in the month before their dissection and in the month prior to follow-up. In addition, we asked whether the patient needed help from their proxies to answer the questions.

\section{Statistical analysis}

Statistical analysis was performed using SPSS 13 for Macintosh statistical software (SPSS Inc.๑), 2001). Demographic data are given as mean values. The NIHSS score is given as a median value. Correlation between functional outcome and QOL was measured with non-parametric correlations (Kendall's tau). Continuous variables were compared with the t-test for normal or the Mann-Whitney $U$ test for abnormal distributed variables. Categorical variables were compared with $\chi^{2}$ and Fisher's exact test as appropriate. QOL of all patients and subgroups (patients with ICAD and VAD; survivors with the following presenting symptoms: ischemic stroke, those with isolated local symptoms or transient ischemic symptoms, those with no or minimal disabilities [mRS 0 or 1] and those with severe disabilities [mRS 2 to 5] at follow-up), were compared with the self-rated QOL before dissection in all patients. Before performing the multivariate regression analysis, a univariate analysis was performed. Variables available at hospital admission were tested one by one against the dependent variable, and variables without association $(p>0.2)$ were removed from the model. The dependent variable was QOL, dichotomized as mentioned above. Then logistic regression analysis with the forward stepwise method was performed to determine the independent association of QOL with other clinical factors. A two sided $\mathrm{p}$-value $<0.05$ was considered statistically significant. 


\section{Results}

\section{Baseline data}

From January 1997 to September 2005, 114 consecutive patients with a SCAD were hospitalized in our stroke center. Six patients died within the first 3 months. Baseline characteristics of 108 survivors ( 50 women, 58 men; mean age 46 years) are shown in Table 1 . Eighty-seven patients had an ICAD, 19 a VAD and 2 a dissection of both vessels. Twenty-eight patients presented with transient or local symptoms, and 80 patients had an ischemic stroke or a retinal infarction. The 108 survivors were contacted after a mean of 1498 days (range 379-3455). Ninety-eight of the 108 survivors $(91 \%)$ were contacted by phone, 9 patients were lost to follow-up and one patient returned the questionnaire, but could not be reached by phone. Outcome at follow-up, measured with the $\mathrm{mRS}$, is given in Table 2 .

\section{QOL before and after SCAD}

The SS-QOL questionnaire was completed by 99 of the 108 survivors (92\%). Seventy-four survivors (75\%) were able to complete the questionnaires without help. SSQOL before dissection was rated as good in 92 of 99 survivors $(93 \%)$, after dissection in 53 patients (54\%). Compared to the self-rated QOL of all patients before dissection, SS-QOL was significantly lower after SCAD $(p<0.001)$ : mean SS-QOL before dissection was 4.63 (95\% confidence interval (CI) 4.55-4.71) and 3.87 (95\% CI 3.69-4.05) after dissection (Fig.1).

\section{QOL and functional outcome (mRS)}

There was an overall correlation of high mRS and impaired QOL $(\mathrm{p}<0.001)$ : patients with a high mRS score were more likely to suffer from an impaired QOL (Kendall's tau $>0.5)$. Nevertheless, 21 of 71 patients $(30 \%)$ with a favorable outcome (mRS 0-1) reported an impaired QOL. Only 2 of 27 patients (7\%) with an unfavorable outcome (mRS 2-5) experienced a good QOL.

\section{Subgroup analysis}

SS-QOL varied among subgroups (Fig. 1). QOL was best in survivors with transient or local symptoms and worst in disabled patients due to stroke (mRS 2-5). A significantly impaired QQL compared to the overall QOL before dissection was not only observed in patients with an ischemic stroke $(\mathrm{p}<0.001)$, but also in patients with isolated local or transient ischemic symptoms $(p<0.038)$ and in patients without a disability $(\mathrm{mRS}=0-1)$ at fol-
Table 1 Baseline characteristics of survivors $(n=108)$

\begin{tabular}{ll}
\hline Variable & \\
\hline Age at symptom onset, mean, (SD; range) & 46 years $(9 ; 18-78)$ \\
Female, $n$ (\%) & $50(46 \%)$ \\
Baseline NIHSS score, median (range) & $4(0-31)$ \\
Time from symptom onset to diagnosis, median (range) & 2 days (0-32) \\
Affected vessel & \\
Carotid artery dissections, $n$ (\%) & $87(80 \%)$ \\
$\quad$ Bilateral, $n$ & 3 \\
Vertebral artery dissections, $n$ (\%) & $19(18 \%)$ \\
$\quad$ Bilateral, $n$ & 1 \\
Carotid and vertebral artery dissections, $n(\%)$ & $2(2 \%)$ \\
Presenting clinical findings on hospital admission, $\mathrm{n}(\%)$ & \\
Ischemic stroke & $79(73 \%)$ \\
TIA & $11(10 \%)$ \\
Retinal infarction & $1(1 \%)$ \\
Local symptoms only & $17(16 \%)$ \\
Other symtpoms at hospital admission, $\mathrm{n}(\%)$ & \\
Headache & $79(73 \%)$ \\
Neck pain & $29(27 \%)$ \\
Horner's syndrome & $42(38 \%)$ \\
Tinnitus & $9(8 \%)$ \\
Cranial nerve involvement & $2(2 \%)$ \\
Risk factors, $n$ (\%) & \\
Hypercholesterolemia & $36(33 \%)$ \\
Current smoking & $26(24 \%)$ \\
Diabetes mellitus & $2(2 \%)$ \\
Hypertension & $26(24 \%)$ \\
Family history of stroke & $16(15 \%)$ \\
History of migraine & $25(23 \%)$ \\
History of preceding minor trauma & $16(15 \%)$ \\
\hline & \\
& \\
\hline
\end{tabular}

Table 2 Outcome

\begin{tabular}{lc}
\hline \multicolumn{2}{l}{ Modified Rankin Scale } \\
\hline All (CAD and VAD), $\mathrm{n}=108$ & $71(66 \%)$ \\
$\quad$ mRS 0-1, $\mathrm{n}(\%)$ & $27(25 \%)$ \\
mRS 2-5, $\mathrm{n}(\%)$ & $9(8 \%)$ \\
Lost to follow-up, $\mathrm{n}(\%)$ & $1(1 \%)$ \\
$\quad$ Missing data, $\mathrm{n}(\%)$ & \\
Carotid artery dissection, $\mathrm{n}=87$ & $56(65 \%)$ \\
mRS 0-1, $\mathrm{n}(\%)$ & $22(25 \%)$ \\
mRS 2-5, $\mathrm{n}(\%)$ & $1(1 \%)$ \\
Missing data, $\mathrm{n}(\%)$ & $8(9 \%)$ \\
Lost to follow-up, $\mathrm{n}(\%)$ & $14(74 \%)$ \\
Vertebral artery dissection, $\mathrm{n}=19$ & $5(26 \%)$ \\
$\quad$ mRS 0-1, $\mathrm{n}(\%)$ & 1 \\
mRS 2-5, $\mathrm{n}(\%)$ & \\
Carotid and vertebral artery dissection, $\mathrm{n}=2$ & \\
$\quad$ Lost to follow-up, $\mathrm{n}$ &
\end{tabular}

low-up ( $\mathrm{p}=0.013)$. SS-QOL was similar in patients with VAD (mean: 3.74, SD: 1.13) and ICAD (mean: 3.92, SD: $0.85)(p>0.05)$. Patients with stroke and with local or 


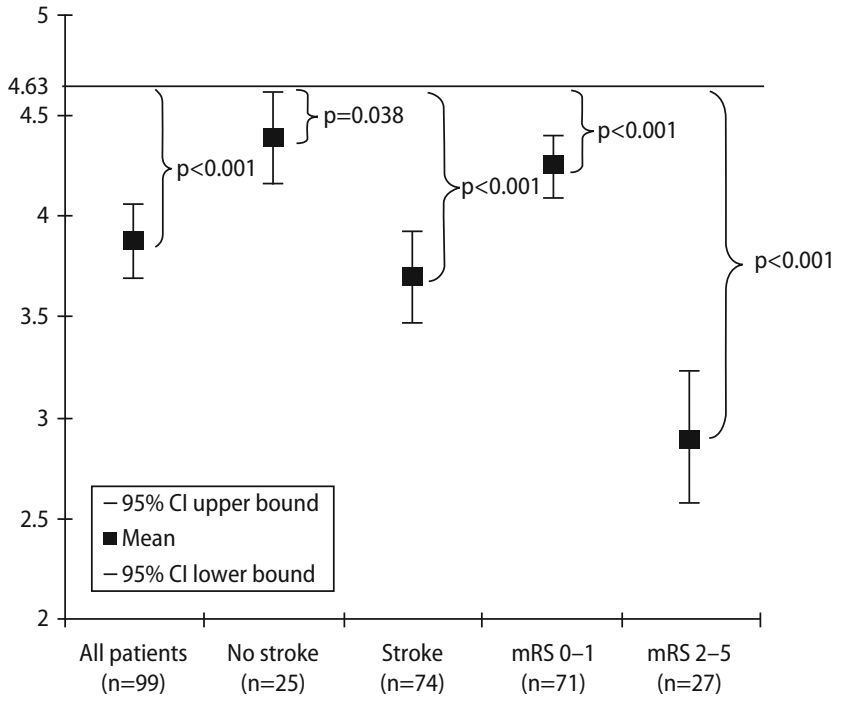

Fig. 1 SS-QOL in survivors after spontaneous cervical artery dissection (SCAD). SS-QOL ranges from 1 to 5 with higher scores indicating better function. The black bar (4.63) shows the mean value of SS-QOL in all patients before their SCAD. CI confidence interval; $p p$ value (A two sided $p$-value $<0.05$ was considered statistically significant); $m R S$ modified Rankin Scale; Stroke all patients with ischemic stroke and or retinal ischemia; No Stroke all patients with a transient ischemic attack or local symptoms

transient symptoms did not differ regarding sex, vascular risk factors and time to follow-up ( $\mathrm{p}>0.05)$.

\section{Subitems of QOL}

Figs. 2 and 3 show the different items of the SS-QOL in patients before and after dissection. The biggest change in QOL in survivors without significant disabilities at follow-up (mRS 0-1) could be observed in the items "thinking", "energy" and "language", whereas in the domains "self care", "personality" and "mood" the smallest change could be assessed. In patients with local or transient symptoms QOL decreased most in the domains "thinking","energy" and "personality", whereas "mood", "family roles", "self-care", "social roles" and "upper extremity function" were hardly impaired.

\section{Residential status, occupation and marital status}

At follow-up, 86 patients ( $88 \%$ ) were living in their home without help, $10(10 \%)$ with help and $2(2 \%)$ in a nursing home. 81 survivors were able to communicate via phone. Before sCAD, 90 patients had been working full time, 8 part-time. After SCAD, 45 survivors had resumed full-time employment, 24 part-time, 2 were retired, 2 were unemployed and 24 were no longer able to work because of their handicap. Marital status did not change significantly before and after SCAD: one patient married after her sCAD.

\section{Predictors of Q0L}

In a multivariate logistic regression analysis, higher NIHSS score on admission (odds ratio (OR): $0.853 ; 95 \%$ confidence interval $(\mathrm{CI}): 0.788-0.922 ; \mathrm{p}<0.001)$ and higher age (OR: 0.914; CI: $0.860-0.971 ; \mathrm{p}=0.003$ ) were independent predictors of an impaired QOL. Time interval from symptom onset to diagnosis $(p=0.249)$, minor trauma before dissection $(\mathrm{p}=0.435)$, cephalgia $(p=0.360)$, cervicalgia $(p=0.188)$, tinnitus $(p=0.260)$ and Horner's syndrome $(\mathrm{p}=0.114)$ were not indepen-
Fig. 2 SS-QOL in patients before spontaneous cervical artery dissection (SCAD) and in survivors with a favorable ( $\mathrm{mRS} 0-1$ ) and an unfavorable (mRS 2-5) outcome. SS-QOL ranges from 1 to 5 with higher scores indicating better function. $m R S$ modified Rankin Scale; Stroke all patients with ischemic stroke and or retinal ischemia; No Stroke all patients with a transient ischemic attack or local symptoms; UE function upper extremity function

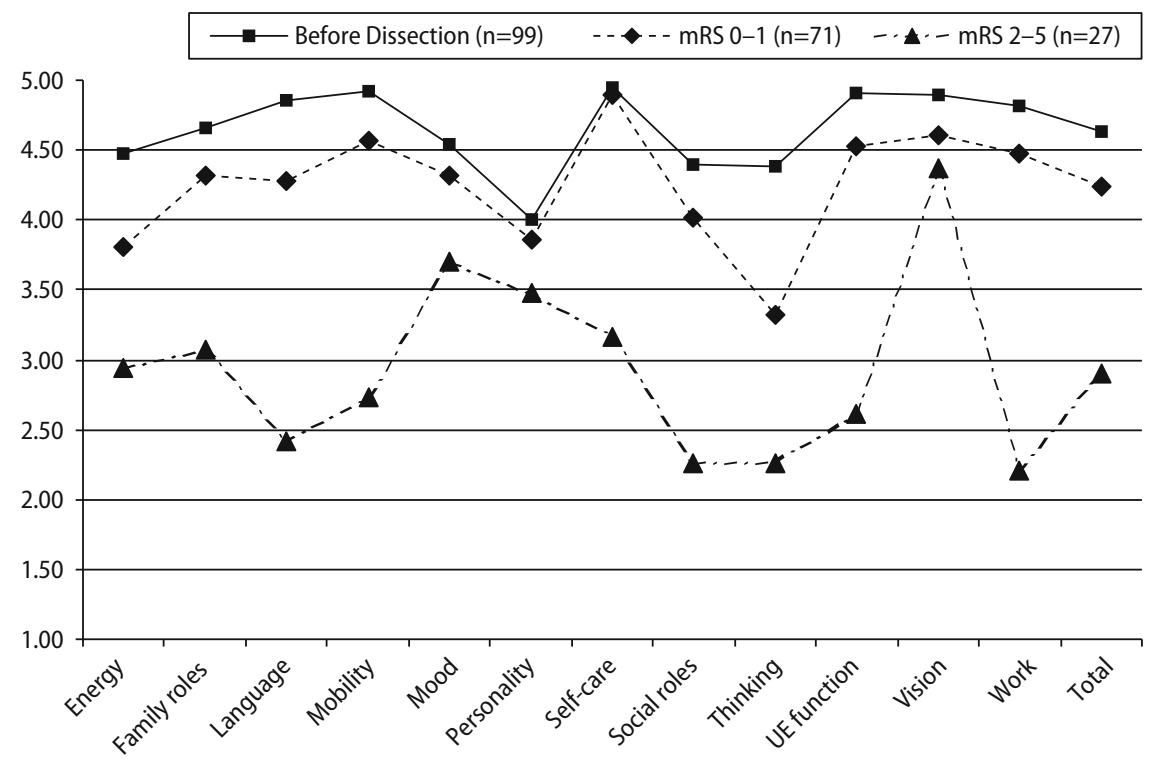


Fig. 3 SS-QOL in patients before spontaneous cervical artery dissection (SCAD) and in survivors with and without stroke. SS-QOL ranges from 1 to 5 with higher scores indicating better function. $m R S$ modified Rankin Scale; Stroke all patients with ischemic stroke and or retinal ischemia; No Stroke all patients with a transient ischemic attack or local symptoms; UE function upper extremity function

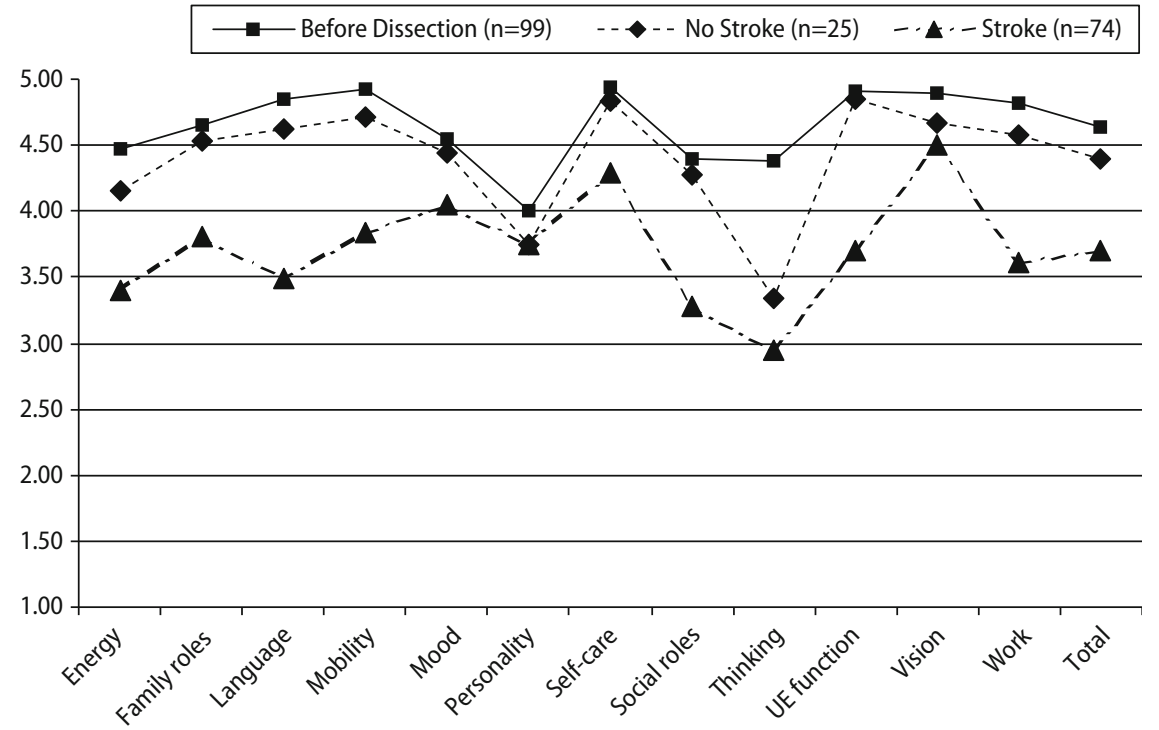

dently associated with QOL in the multivariate analysis.

\section{Discussion}

In this study we analyzed QOL and long-term functional outcome in survivors after SCAD. After a mean follow-up of 4 years, $72 \%$ of the patients had no significant functional disabilities (mRS $0-1$ ). Patients' opinion of outcome differed significantly from the functional outcome assessed with the mRS. When using QOL measures only $54 \%$ of all survivors reported a good QOL (SS-QOL $\geq 4$ ). This is the main finding of our study analyzing functional outcome and QOL in all survivors after SCAD.

In general, QOL was worse in handicapped patients than in those without significant disabilities, a fact already previously shown in other patients with stroke [17]. However, when compared to the premorbid condition of all patients, QOL was not only impaired in the disabled, but also in those without significant disabilities at follow-up and in survivors without an ischemic stroke. This interesting finding reflects our clinical observation that some of these patients are complaining of discomfort even years after their dissection despite no obvious physical abnormalities. sCAD affects mostly young and previously healthy patients, who are not expecting such an incident, which is often experienced as a life-threatening event. Survivors are concerned about suffering a second dissection, even when aware of the low recurrence rate and the generally favorable outcome $[5,18]$. The lack of a convincing scientific explanation for the pathogenesis and etiology of sCAD worries some patients. In contrast to a stroke due to atherosclerosis, the chance of having a SCAD is not associated with vascular risk factors and hence is not predictable. There- fore, primary and secondary prevention measures are scarce and patients cannot influence their destiny.

Domains of the SS-QOL that were mostly affected in survivors without significant disabilities at follow-up or without a stroke were nonspecific items such as "energy" and "thinking". This may reflect the patient's general concern and represent a psychological phenomenon, because physical function-related items such as "selfcare", "social roles", "mobility" and "upper extremity function" were barely affected. The item "mood" was not affected. Therefore, the deterioration in QOL is unlikely to be due to major depression, a common important contributing factor of an impaired QOL.

The most appropriate end-point in follow-up studies after SCAD is still under debate: recurrence rate is low; lack of complete morphological recovery of the affected vessel does not seem to be an appropriate outcome measure unless it is accompanied by an ischemic stroke, and traditional functional outcome measurements such as mRS show ceiling effects [19]. Half of our patients had a mRS of 0 and $72 \%$ a mRS of 0 or 1 . QOL scales on the other hand measure not only functional outcomes but also assess psychological and cognitive functions like memory, emotion, thinking, communication and social role. In the present study, QOL was more sensitive than traditional outcome measures and was able to assess sequelae in patients with local symptoms only and in those without functional deficits at follow-up. Previous studies of QOL in patients after sCAD are scarce: to our knowledge, QOL has never been assessed in patients after carotid artery dissections and in patients with sCAD without concomitant ischemic events. To date, there is only one retrospective study of 30 patients with a stroke due to VAD: Czechowsky et al. found a poorer outcome on QOL measures than on standard stroke scales, which is in line with our results [20]. 
In a multivariate regression analysis, high NIHSS score on admission and higher age were independent predictors of impaired QOL. In a previous study of 195 survivors after VAD low baseline NIHSS and younger age were independent predictors of a favorable functional outcome (mRS 0-1) [21]. Because of the correlation of $\mathrm{mRS}$ and QOL our results are in line with these data.

Our study has several limitations. A total of $25 \%$ of survivors were unable to complete the questionnaire without help. Patients' perception of QOL and caregivers' perception may vary $[22,23]$. However only $8 \%$ without a stroke and $8 \%$ without significant disability needed help by their proxies. Therefore, it seems unlikely that these results might have substantially influenced our results.

Another limitation is the wide range of time to follow-up inquiry. However all patients had at least one year of follow-up. Because most of the changes after a stroke occur within a few months and changes after years are usually small, it is unlikely that this might have biased our results. Thomassen et al. showed in an analysis, assessing mRS 3, 6 and 12 months after intravenous thrombolysis that major improvement does not occur between 3 to 12 months [24]. Outcome in 173 patients after intraarterial thrombolysis of our own cohort was sustained two years after thrombolysis and major improvement between 3 months and 2 years was not observed [25]. Potentially, new and/or other diseases or life conditions might have influenced QOL in our patients because of the wide range of time to follow-up. However, time to follow-up did not differ significantly in patients with and without stroke as well as in patients with $\mathrm{mRS}$ $0-1$ and $2-5$. Further analyses such as stratification according to time to follow-up, were not sensible because of the rather small sample size of our study. Furthermore, QOL was related to the functional status, which should have decreased in the presence of a severe disabling disease. Moreover marital status - an important factor that can influence QOL - did not change in our patients.

Survivors had to rate their QOL before dissection: in retrospect, the past might have been perceived more pleasantly than the present, as unpleasant conditions may have been forgotten. Patients with major disabilities might rate their QOL before their SCAD significantly higher and therefore may overestimate the impact of the sCAD on their QOL, whereas patients with little disabilities might rate their QOL before dissection too low. However, even if patients with no significant functional disabilities had underestimated their QOL before their SCAD in the present analysis, QOL was still significantly lower than before the SCAD. Assessing QOL before dissection is impossible and population-based values of SSQOL to compare our patients' condition with healthy controls are not available. Therefore, mean SS-QOL in all patients before their dissection was assumed as best available measure for a good QOL. There was no difference in rating premorbid QOL by proxies and patients and $95 \%$ CI in SS-QOL before dissection was very low.

This study may provide a basis for further QOL research in patients with $\mathrm{SCAD}$. QOL should be assessed in all prospective studies, analyzing outcome in patients with SCAD and should be compared with healthy controls. Mediator variables such as coping behavior, response shift, benefit finding and aspects of individual QOL assessment should be focused in further studies.

In conclusion QOL is significantly impaired in longterm survivors after $\mathrm{SCAD}$, even in patients with local or transient symptoms only and in patients without functional disability. QOL is more sensitive than traditional outcome scales to assess sequelae after sCAD and deserves attention as an outcome measure in these patients.

Conflict of interest The authors declare no conflict of interest.

Acknowledgments We thank Pietro Ballinari for statistical advice.

\section{References}

1. Leys D, Bandu L, Hénon H, MounierVehier F, Rondepierre P, Godefroy O (2002) Clinical outcome in 287 consecutive young adults (15 to 45 years) with ischemic stroke. Neurology 59:26-33

2. Biousse V, D'Anglejan-Chatillon J, Touboul PJ, Amarenco P, Bousser MG (1995) Time course of symptoms in extracranial carotid artery dissections. A series of 80 patients. Stroke 26: 235-239
3. Arnold M, Cumurciuc R, Stapf C, Favrole P, Berthet $\mathrm{K}$, Bousser MG (2006) Pain as the only symptom of cervical artery dissection. J Neurol Neurosurg Psychiatry 77:1021-1024

4. Arauz A, Hoyos L, Espinoza C, Cantu C, Barinagarrementeria F, Roman $\mathrm{G}$ (2006) Dissection of cervical arteries: long-term follow-up study of 130 consecutive cases. Cerebrovasc Dis 22:150-154

5. Lee VH, Brown RD, Mandrekar JN, Mokri B (2006) Incidence and outcome of cervical artery dissection: A population-based study. Neurology 67: 1809-1812
6. Kasner SE, Hankins LL, Bratina P, Morgenstern LB (1997) Magnetic resonance angiography demonstrates vascular healing of carotid and vertebral artery dissections. Stroke 28:1993-1997

7. Auer A, Felber S, Schmidauer C, Waldenberger P, Aichner F (1998) Magnetic resonance angiographic and clinical features of extracranial vertebral artery dissection. J Neurol Neurosurg Psychiatry 64:474-481

8. Provenzale JM, Morgenlander JC, Gress D (1996) Spontaneous vertebral dissection: clinical, conventional angiographic, CT, and MRI findings. J Comput Assist Tomogr 20:185-193 
9. Mokri B (1990) Traumatic and spontaneous extracranial internal carotid artery dissections. J Neurol 237: 356-361

10. Baumgartner RW, Arnold M, Baumgartner I, Mosso M, Gönner F, Studer A, Schroth G, Schuknecht B, Sturzenegger M (2001) Carotid dissection with and without ischemic events. Neurology $57: 827-832$

11. Headache Classification Committee of the International Headache Society (1988) Classification and diagnostic criteria for headache disorders, cranial neuralgias and facial pain. Cephalalgia 8(Suppl 7):1-96

12. International Classification of Headache Disorders, $2^{\text {nd }}$ edition (2004) Cephalalgia24(Suppl 1):1-160

13. Brott T, Adams HP Jr, Olinger CP, Marler JR, Barsan WG, Biller J, Spilker J, Holleran R, Eberle R, Hertzberg V, Roorick M, Moomaw CJ, Walker M (1989) Measurements of acute cerebral infarction: a clinical examination scale. Stroke 20:846-870

14. Williams LS, Weinberger M, Harris LE, Clark DO, Biller J (1999) Development of a Stroke-Specific Quality of Life Scale. Stroke 30:1362-1369
15. Van Swieten JC, Koudstaal PJ, Visser MC, Schouten HJ, van Gijn J (1988) Interobserver agreement for the assessment of handicap in stroke patients. Stroke 19:604-607

16. Merino JG, Lattimore SU, Warach S (2005) Telephone assessment of stroke outcome is reliable. Stroke 36:232-233

17. Fischer U, Anca D, Arnold M, Nedeltchev K, Kappeler L, Ballinari P, Schroth G, Mattle HP (2008) Quality of life in stroke survivors after local intraarterial thrombolysis. Cerebrovasc Dis 25(5):438-444

18. Bassetti C, Caruzzo A, Sturzenegger M, Tuncdogan E (1996) Recurrence of cervical artery dissection. A prospective study of 81 patients. Stroke 27: 1804-1807

19. Leys D, Debette S (2006) Long-term outcome in patients with cervicalartery dissections: there is still a lot to know. Cerebrovasc Dis 22:215

20. Czechowsky D, Hill MD (2002) Neurological outcome and quality of life after stroke due to vertebral artery dissection. Cerebrovasc Dis 13:192-197
21. Arnold M, Bousser MG, Fahrni G, Fischer U, Georgiadis D, Gandjour J, Benninger D, Sturzenegger M, Mattle HP, Baumgartner RW (2006) Vertebral artery dissection. Presenting findings and predictors of outcome. Stroke 37: 2499-2503

22. Dorman PJ, Waddell F, Slattery J, Dennis M, Sandercock P (1997) Are Proxy assessments of health status after stroke with the EuroQol questionnaire feasible, accurate, and unbiased? Stroke 28:1883-1887

23. Williams LS, Bakas T, Brizendine E, Plue L, Tu W, Hendrie H, Kroenke K (2006) How valid are family proxy assessments of stroke patients' healthrelated quality of life? Stroke 37: 2081-2085

24. Thomassen L, Waje-Andreassen U, Naess H, Elvik MK, Russell D (2005) Long-term effect of intravenous thrombolytic therapy in acute stroke: responder analysis versus uniform analysis of excellent outcome. Cerebrovasc $D$ is 20:470-474

25. Nedeltchev K, Fischer U, Arnold M, Ballinari P, Haefeli T, Kappeler L, Brekenfeld C, Remonda L, Schroth G, Heinrich PM (2006) Long-term effect of intraarterial thrombolysis in stroke. Stroke 37:3002-3007 